Instituto Internacional de Investigación y Desarrollo Tecnológico Educativo INDTEC, C.A.

DOI: https://doi.org/10.29394/Scientific.issn.2542-2987.2021.6.19.17.329-348

OAI-PMH: http://www.indteca.com/ojs/index.php/Revista Scientific/oai

Ensayo Original / Original Essay

\title{
Educación emocional un complemento en el proceso enseñanza- aprendizaje virtual a nivel superior durante COVID-19
}

\author{
Autor: Diego Alvarado Melitón \\ Hospital Psiquiátrico Fray Bernardino Álvarez, HPFBA \\ ocupacional fray@outlook.com \\ Ciudad de México, México \\ https://orcid.org/0000-0002-4116-885X
}

Resumen

La educación emocional es un complemento que permite hacer frente a situaciones adversas como las que se viven actualmente en esta pandemia de COVID-19, a nivel de educación superior, es importante la formación académica de los estudiantes, así como adquirir competencias emocionales usadas en el entorno académico, social y laboral mediante estrategias justificadas para favorecer el proceso de enseñanza-aprendizaje virtual, teniendo como objetivo principal que el docente digital tenga un reto para adaptar estrategias didácticas innovadoras, habilidades y destrezas emocionales, sin dejar de lado el conocimiento teórico-practico impartido a los alumnos en las aulas virtuales, así como la socialización interactiva basada en un aprendizaje socioemocional con un repertorio de habilidades emocionales que favorece al universitario y que estas puedan ser y hacer frente a cualquier situación adversa, evitando el aislamiento social académico y afrontando emociones negativas que propicia el estar demasiadas horas frente a un monitor por parte de los actores de la educación.

\section{Palabras clave: educación; enseñanza; docente; pandemia; aislamiento social.}

Cómo citar este ensayo:

Alvarado, D. (2021). Educación emocional un complemento en el proceso enseñanzaaprendizaje virtual a nivel superior durante COVID-19. Revista Scientific, 6(19), 329-348, e-ISSN: 2542-2987. Recuperado de: https://doi.org/10.29394/Scientific.issn.2542-2987.2021.6.19.17.329-348

Fecha de Recepción: 02-09-2020
Fecha de Aceptación: 25-12-2020
Fecha de Publicación: 05-02-2021 


\title{
Emotional education a complement in the virtual teaching-learning process at a higher level during COVID-19
}

\begin{abstract}
Emotional education is a complement that allows facing adverse situations such as those currently experienced in this COVID-19 pandemic, at the higher education level, the academic training of students is important, as well as acquiring emotional skills used in the academic, social and work environment through justified strategies to favor the virtual teaching-learning process, with the main objective that the digital teacher has a challenge to adapt innovative didactic strategies, emotional abilities and skills, without neglecting theoretical-practical knowledge taught to students in virtual classrooms, as well as interactive socialization based on socio-emotional learning with a repertoire of emotional skills that favors the university and that these can be and face any adverse situation, avoiding academic social isolation and facing emotions negative that favors being too many hours in front of a monitor by educational actors.
\end{abstract}

Keywords: education; teaching; teacher; pandemic; social isolation.

How to cite this essay:

Alvarado, D. (2021). Emotional education a complement in the virtual teaching-learning process at a higher level during COVID-19. Revista Scientific, 6(19), 329-348, e-ISSN: 2542-2987. Recovered from: https://doi.org/10.29394/Scientific.issn.2542-2987.2021.6.19.17.329-348

Date Received: 02-09-2020
Date Acceptance: 25-12-2020
Date Publication: 05-02-2021 


\section{Ensayo Original / Original Essay}

\section{Introducción}

El presente trabajo tiene como finalidad dar a conocer la importancia de la aplicación de la educación emocional y la inteligencia emocional en el nivel de educación superior en México durante la pandemia por COVID-19, por lo cual se retoman algunos representantes de estas teorías que se usan para este ensayo como lo son las presentadas en las investigaciones de Bisquerra y García (2018a); Palomares-Ruiz y Serrano-Marugán (2016a); y Nadal (2015a): siendo de importancia promover y aplicar competencias emocionales en la práctica del estudiante universitario durante el aislamiento social y favoreciendo una regulación emocional durante el proceso de enseñanza aprendizaje virtual, además de promover una relación empática entre profesorado y alumno, creando una estabilidad emocional al implementar estrategias didácticas que se adapten a esta situación sanitaria.

Estas estrategias didácticas fundamentadas en los saberes del conocimiento deberán cumplir con las exigencias a nivel superior, tomando en cuenta las características emocionales de los alumnos universitarios que poseen y el cual puede beneficiar en el proceso de enseñanza-aprendizaje virtual, mediante el uso de las plataformas educativas, pero sin dejar de lado las evaluaciones, ante este cambio tan drástico por un aula virtual, es algo complicado obtener resultados medibles en el nivel académico y emocional tanto del profesorado y del alumno, se tiene que buscar la manera de demostrar que se está cumpliendo un objetivo específico en la adquisición del conocimiento desarrollando habilidades y destrezas emocionales que permitan afrontar la situación actual y futura.

Por lo tanto, se habla sobre los beneficios que se pueden obtener al complementar la educación emocional a nivel de educación superior afrontando el aislamiento social derivado de la pandemia de COVID-19 y las emociones negativas que trae consigo y la posibilidad de implementar estrategias de afrontamiento emocional durante las clases virtuales impartidas 


\section{Ensayo Original / Original Essay}

en las plataformas educativas donde se implementa el proceso de enseñanzaaprendizaje virtual.

Posteriormente, el objetivo principal es que el docente virtual deberá ser una adaptación a la era tecnológica y virtual de esta manera se podrá trasmitir esos conocimientos, aplicando estrategias innovadoras y fomentando habilidades y destrezas emocionales a sus alumnos de grado universitario, siendo un complemento emocional a su formación académica.

\section{Desarrollo}

Para dar inicio al presente ensayo que va dirigido a la educación emocional como un complemento ante la situación de COVID-19 a nivel superior en México, siendo un tema de poca relevancia o simplemente no se le da una prioridad en los distintos niveles de educación superior en México y sin dejar de lado que nuestros formadores docentes al ser instruidos en un modelo tradicionalista, carecen o son pocas las habilidades a desarrollar en el proceso de enseñanza aprendizaje, cuya finalidad actual es la de dar una importancia necesaria para poner en práctica la educación emocional ante los nuevos procesos de enseñanza-aprendizaje virtuales.

Teniendo en cuenta que son de suma importancia, debido a la integración de competencias emocionales, aunque es una tarea difícil para adaptarse a la modificación de nivel superior, por parte del docente la dificultad ante las nuevas estrategias didácticas implementando plataformas virtuales debido a la pandemia de COVID-19, como se sabe este virus ha causado varias repercusiones en sectores como lo es alojamiento, esparcimiento, comercio, transporte, salud y educación, ahora bien, para hacer frente ante tal situación adversa, se recurre al empleo de tecnologías educativas que cuentan con varios recursos didácticos para facilitar las clases y teniendo como objetivo reemplazar el método presencial.

Aunque se sabe que esto puede afectar de una manera significativa a 
la educación en sus diversos niveles de formación académica, resaltando que es elemental poner en práctica ciertas habilidades emocionales en un entorno físico para disminuir o afrontar esta situación, como lo menciona el Banco Mundial, World Bank (2020):

El doble impacto del cierre de las escuelas y de la recesión mundial podría tener costos a largo plazo para la educación y el desarrollo si los gobiernos no reaccionan con rapidez para contrarrestarlos. El cierre de escuelas provocará una pérdida de aprendizajes, un aumento en la cantidad de deserciones escolares y una mayor inequidad; la crisis económica, que afecta a los hogares, agravará el daño, pues vendrá acompañada de menor oferta y demanda educativa [...] (pág. $5)$.

Es difícil conseguir una adaptación a estos nuevos elementos tecnológicos, tanto para profesores y estudiantes que suelen ser usados frecuentemente, por lo que se observa cotidianamente al emplearlos como recursos educativos, tener poca experiencia involucrando un grado de complejidad alto y otros factores como lo es las pocas o nulas actualizaciones que se imparten a los docentes sobre el manejo de estas plataformas y que en algunas instituciones no se le brinda la atención necesaria ni la constante capacitación, asesoría y actualización de estos recursos tecnológicos para el uso del docente.

Del mismo modo, presenta problemas al hacer uso de estas plataformas, causa una serie de emociones negativas como lo son el estrés, enojo, tristeza, frustración, miedo, ansiedad y no hay una manera adecuada para su regulación emocional, por lo que se recurre a tomar conductas inadecuadas que al cabo de un periodo estas repercuten a nivel personal, familiar y social, teniendo en cuenta otro factor como la economía de las familias mexicanas no es suficiente para lograr terminar los estudios universitarios, quien para los alumnos deciden recurrir a una deserción estudiantil, mostrando emociones negativas ya mencionadas. 
Cabe destacar que la educación emocional es una importante estrategia que debe complementarse a niveles básicos para así dar soluciones ideales ante situaciones que se viven actualmente, como lo manifiesta Soler, Aparicio, Díaz, Escolano y Rodríguez (2016):

Por tanto, es urgente poner en marcha un proceso de enseñanza-aprendizaje centrado «no solo en la "parte" cognitiva, sino también en la emocional y social». El sistema educativo actual deberá responder a las necesidades de la comunidad educativa del presente y de las que se prevén en el futuro (pág. 11).

Además se debe responder a las necesidades actuales de educación y su evolución de aulas físicas por las aulas virtuales, en el cual se debe aplicar un intercambio constante de conocimiento teórico-practico haciendo uso de herramientas tecnológicas al que vamos a llamar, proceso de enseñanza aprendizaje virtual, haciendo uso de estrategias didácticas adaptadas mediante habilidades sociales generalizadas en los alumnos y de las cuales habría que sacar mayor provecho en las clases en línea, y obtener un aprovechamiento de este conocimiento que se le brinda al estudiante universitario, sustentados mediante el modelo de competencias emocionales cuya relación debe ir de la mano con los pilares de la educación.

Al poner en marcha un modelo de competencias e inteligencia emocionales a través de estrategias didácticas que se pondrán a prueba desde una clase virtual, estás tendrán como finalidad favorecer un desarrollo personal y profesional a través del transcurso de la formación educativa y de la interacción virtual mediante los actores de la educación, así como lo señala Goleman (1995), citado por Palomares-Ruiz y Serrano-Marugán (2016b):

La inteligencia emocional es una forma de interactuar con el mundo, valora los sentimientos y engloba habilidades como el control de los impulsos, la autoconciencia, la motivación, el entusiasmo, la perseverancia, la empatía, la agilidad mental, etc. que configuraran rasgos de carácter, como la 
autodisciplina, la compasión o el altruismo, indispensables para una buena y creativa adaptación social. Asimismo, este autor, considera la competencia emocional como la capacidad para reconocer nuestros propios sentimientos y los ajenos, con capacidad para manejar de manera positiva las emociones, especialmente aquellas que tienen que ver con las relaciones humanas (pág. 26).

La educación emocional permite al docente y estudiante adquirir habilidades que favorecen su educación emocional y maximizar sus procesos de aprendizaje afrontando situaciones que demanda la actualidad académica, personal y laboral que se le presenten día a día dando soluciones optimas y de manera más adecuada a las diferentes disciplinas como lo son la ciencia, tecnología, ingeniería, en cambio para el profesorado se hace evidente un mayor dominio en la capacidad de dominar sus pensamientos y acciones dentro y fuera del aula, retomando que debido a este riesgo sociosanitario por el que estamos atravesando se hace uso de plataformas educativas.

Queda claro que a pesar de tener los recursos necesarios para seguir impartiendo el conocimiento, se viven momentos de tecnoestrés, conflictuando las emociones del docente y del estudiante, a tal grado de perder la conciencia emocional de ambos actores, aunque al implementar procesos de enseñanzaaprendizaje virtuales la educación que enmarque estas emociones se pretende lograr una adaptación y procesamiento emocional que va permitir una mejor toma de decisiones y manejo adecuado de situaciones que se viven actualmente, de acuerdo con Bisquerra y García (2018b):

La educación emocional es una de las innovaciones psicopedagógicas de los últimos años que se propone responder a algunas de las necesidades sociales que no quedan suficientemente atendidas en las materias académicas ordinarias (STEM). Se propone optimizar el desarrollo humano. Es decir, el desarrollo integral de la persona (desarrollo físico, intelectual, moral, social, emocional, etc.) (pág. 16). 
A pesar de hacer énfasis en las competencias a nivel superior en educación y su implementación en la práctica hacia los modelos educativos en beneficio de los estudiantes, las instituciones tienen poca relevancia sobre educación emocional, tomando en cuenta que es esencial brindar una enseñanza emocional en las diferentes asignaturas y que al combinar con competencias emocionales se puede asociar nuevos conocimientos y que este sea transversal a dichas áreas de aplicación laboral.

Esto permite tener un desempeño adecuado, en el cual se observe los objetivos planteados en su plan académico, por otro lado, también se vería reflejado que las habilidades emocionales como, la empatía y la habilidad social, se refuerzan o tienen mayor predominio al tomar decisiones que sean acorde a las necesidades actuales y situaciones emergentes y de su entorno laboral, personal y social, además del objetivo principal que es aplicar conocimientos actuales y ligados a demandas del contexto laboral, haciendo uso el estudiante de su inteligencia racional, sin embargo, los estudiantes son más propensos a enfocarse a cumplir con la entrega de tareas, estudiar para exámenes, entre otros factores relevantes, lo que conlleva a tener un conflicto emocional que desencadena problemas de salud física y mental observado mediante emociones negativas y llegando a la deserción educativa.

La transmisión de un conocimiento virtual es esencial en nuestros días y que ahora es parte del proceso de enseñanza-aprendizaje en el cual por las diferentes plataformas educativas el docente puede llevar a cabo una mayora socialización virtual, por lo que al momento de estar en línea y con cámara encendida durante la clase el docente puede hacer una simbiosis de emociones compartidas ante los oyentes del aula virtual, para favorecer una autorregulación emocional.

Es importante resaltar que la interacción social favorece relaciones sociales y es esencial primero conocerse a mí mismo para crear este tipo de vínculos afectivos y empáticos ante un grupo determinado en la sociedad y 


\section{Ensayo Original / Original Essay}

ante un aula virtual, ahora bien, en las aulas virtuales se favorece este proceso de socialización cuando se cuentan con los recursos y habilidades adecuados, pero en ocasiones al no contar con estas competencias hay una gran dificultad que se enmarcan y se observan como abordajes digitales poco creativos o no cumplen con un orden determinado, provocando nula atención de los estudiantes y teniendo una clase poca interactiva lo cual no permite crear habilidades sociales y resultado a unos denominados incompetentes sociales.

En efecto, al no tener una situación emocional controlada de nuestra vida en la infancia y en el transcurso de la vida o es poco regulada, es un reto difícil que en las escuelas se le tome la importancia necesaria y ahora tal vez por recurrir a un modelo tradicionalista y al pasar a un entorno virtual los docentes se les ha dificultado la evolución y adaptación a docentes digitales teniendo como principal problema afrontar los nuevos modelos educativos, pero que sin dejar atrás la labor docente que conlleva un compromiso y de mucha responsabilidad que tienen los actores educativos, así lo remarca López (2005):

La educación no es una tarea fácil y menos en las primeras edades en la que los niños y niñas necesitan la ayuda del educador o educadora. No es tan sólo un reto para la escuela educar emocionalmente a los niños, sino que también lo es educar a todos aquellos agentes, tanto maestros, como familiares, monitores, etc., que se encargan de favorecer el crecimiento personal de estos niños y niñas (pág. 155).

Entre otros aspectos, se es consiente que como docente digital ahora en las instituciones educativas se debe cumplir cierto plazo de tiempo para cubrir los planes de estudio y en tan poco tiempo la entrega de su proceso de enseñanza-aprendizaje virtual tomando en cuenta que es más enfocado a la memorización y repetición del conocimiento en los diferentes niveles de educación, teniendo en cuenta que estos se deben justificar mediante los pilares de la educación y como aspecto importante, es aquel donde a nivel 


\section{Ensayo Original / Original Essay}

superior es la de complementar mediante competencias básicas, genéricas y específicas.

Realmente es un tanto complicado, debido a que ya se venía trabajando con estas competencias en un modelo presencial, ahora con este nuevo reto la capacitación universitaria debe ser acorde a las demandas actuales que se viven por la pandemia, si tenemos presente que el conocimiento que va adquirir será aplicado en un contexto social, donde las demandas son muy diferentes en su aplicación teórica y práctica, agregando que si no tiene una regulación emocional y habilidades sociales equilibradas, el estudiante universitario tendrá una mayor dificultad para desenvolverse en un puesto de trabajo y en manejo de su regulación emocional ante situaciones que impliquen la toma de decisiones complejas como lo menciona Suberviola (2020):

El Informe Delors (UNESCO, 1996) reconoce que la Educación Emocional es un complemento indispensable en el desarrollo cognitivo y social, además de una herramienta profiláctica incomparable en los conflictos interpersonales, puesto que muchos problemas tienen su origen en el ámbito emocional. Por lo tanto, una correcta instrucción emocional, no solo va a favorecer a la propia persona, sino que también va a incidir en una sociedad menos violenta y más justa (pág. 190).

Durante la formación académica universitaria se promueve y fundamenta, el saber pensar, saber hacer, saber convivir y saber ser para afrontar problemas que causan conflictos en situaciones reales al estudiante, por parte del docente hay un enfoque más dirigido a trasmitir el conocimiento por el proceso de enseñanza teórico y recurriendo al uso de la memorización y repetición de información, sin darle prioridad a las emociones que se encuentran alrededor de la clase virtual, generando cierto grado de frustración cuando hay que aplicar este conocimiento a situaciones que simulan entornos reales. 


\section{Ensayo Original / Original Essay}

Ahora si el proceso de enseñanza-aprendizaje virtual tiene repercusiones en cuanto a emociones negativas muy bien marcadas en el caso práctico del conocimiento y esto se observa a menudo a nivel superior, tal es el caso del saber convivir, si actualmente se usan diferentes redes sociales cuya finalidad es la de interactuar y formar amistades, pero si este saber es aplicado en plataformas educativas el rechazo de una sana convivencia por lo que la tarea para el docente es un reto desafiante al establecer actividades teóricas y prácticas encaminadas al saber hacer, convivir y ser, entonces como será la repercusión para los estudiantes universitarios, dicho en palabras de Miguel (2020):

Los actores directos del proceso formativo en educación superior se ven rebasados en diferentes ámbitos, en específico, en dos: las condiciones estructurales, como son las tecnológicas, cantidad de dispositivos con los que cuenta, el espacio geográfico y la conectividad a Internet; por otra parte, se encuentran los pertenecientes a los que están en sus posibilidades como personas: los socioemocionales, las nuevas formas de aprender, las competencias digitales, la comunicación y la organización eficaz (pág. 37).

Los docentes digitales se empeñan en realizar planeaciones didácticas que se enfocan al tipo de conocimiento teórico o practico el cual se va a trasmitir, siendo un proceso desafiante que se complica al tener que enfocarlo a objetivos y plazos cortos de tiempo, agregando competencias y demandas actuales de información, tecnología, saberes, etc., el adaptar dichas planeaciones didácticas, conlleva responsabilidad y dedicación a las mismas.

En este contexto, se debe tener como función docente agregar los principios de la educación emocional, estos elementos van a interactuar entre el saber pensar, hacer, convivir y ser, mediante una autoconciencia emocional, si bien es importante mencionar, que, al estar triste, enojado, alegre, entre otras emociones, influye en el aprendizaje que se desea trasmitir, como lo indica, Zepeda-Hernández, Abascal-Mena y López-Ornelas (2015): 
Cada vez que nos enfrentamos a algo desconocido o nuevo, nuestro proceso psicológico mantiene un alerta que conlleva una emoción inmersa, la cual puede ser positiva o negativa. A su vez, los momentos emotivos causan una sociabilidad innata a manera de compartir o enfrentar diversas situaciones (pág. 194).

Entre las competencias emocionales que se deberían fomentar en el alumnado y los docentes a mi criterio personal, es tener conciencia emocional, autonomía, competencias sociales, autorregulación, motivación, empatía y habilidades sociales, debido a que, si uno no sabe que emociones presenta, no tendrá idea de cómo se sienten los demás teniendo en cuenta que ahora es un proceso más difícil al estar en aulas virtuales y que nuestros alumnos tienen diferentes distractores en su proceso de enseñanza-aprendizaje virtualizado.

Por otra parte, la regulación de las emociones, la cual se dirigida a la acción con un pensamiento consiente, no solo dejarse llevar por la emoción en el momento, la autonomía personal por su lado es favorable para lograr una facultad de gobernar las propias decisiones que se toman sin influirse por otros, favoreciendo a formar pensadores críticos, la autorregulación nos permite responder a demandas actuales de un sistema social espontáneamente y permite una mejor percepción de las emociones de otras personas con las que se va rodear el estudiante en un futuro laboral.

La motivación es esencial durante y después de la formación académica debido a que permite encontrar recursos emocionales para lograr metas, perseguirlas y no decaer en el intento, la empatía es algo fundamental porque el docente es quien refleja esta actitud frente a los estudiantes en formación y como modelo a seguir del estudiantes este tendrá la capacidad de entender y compartir sentimientos ajenos, algo que actualmente se está perdiendo, notablemente en los entornos laborales, como ejemplos las autoridades de centros de trabajo que reflejan delirios de omnisapiencia y 


\section{Ensayo Original / Original Essay}

omnipresencia.

Finalmente, las habilidades sociales que permiten un convivir en armonía durante la formación académica y en los entornos futuros de trabajo, en el cual se muestren ciertos principios y valores. Actualmente, al permanecer demasiado tiempo en línea para las clases virtuales hay un desequilibrio reflejado en conflictos interpersonales y posiblemente tomando actitudes violentas al sentirse frustrados por no saber cómo expresar sus emociones al permanecer horas frente a un monitor, llevando a largo plazo a un aislamiento social y no necesariamente en esta pandemia, sino como un hábito que se empieza a formar en los estudiantes universitarios favoreciendo una afección en la conciencia emocional y desatando problemas de salud mental.

Por consiguiente, se hace mención que la regulación emocional es un área que se afecta tanto para docentes y alumnos, por lo que es necesario darle importancia, como lo expresa Jiménez (2020): "nuestra regulación emocional es un conjunto de procesos internos conscientes que nos permiten experimentar emociones sin ser desbordados e inundados por ellas. Nos permiten evaluar y modificar nuestras reacciones para alcanzar nuestras metas y objetivos" (pág. 557).

En este mismo orden de ideas, es importante dar a conocer que las repercusiones de una mala regulación emocional en el docente conllevan a un desequilibrio entre emoción, cognición y su comportamiento, teniendo un mal desempeño en su práctica educativa virtual, por lo que es importante tener un dominio de estas emociones frente al aula virtual, en las cuales no es nada fácil lograrlo, siendo conscientes que el trabajo como docente conlleva a varios procesos de planificación de clases, evaluaciones, evidencias, etc., sin embargo se debe hacer uso de estrategias emocionales que ayuden al docente para hacer frente ante determinadas situaciones que se viven actualmente.

De esta forma, como docente se tendrán herramientas que equilibren 


\section{Ensayo Original / Original Essay}

su estado de ánimo ante la impulsividad y la frustración logrando una mayor tolerancia ante los actuales casos que se viven, por mencionar algunos, como lo es, discutir con alumnos y padres de familia, minorizar los temas de clases por falta de empatía e interés, entre otros problemas.

El docente tiene una función específica para obtener resultados a partir de instrumentos de evaluación emocional, tales como la percepción, asimilación, comprensión y regulación emocional, aunque en nuestro sistema educativo mexicano, se basa más en pruebas de inteligencia, haciendo uso de la memorización de la información, por lo tanto, hay una evidente evaluación dirigida a procesos mecánicos de retención de conocimiento, y en general no hay una comprensión del conocimiento adquirido.

Como es sabido, las inteligencias múltiples son variadas y a su vez estas tienen características particulares así como un proceso que implica la asimilación de la información, haciendo uso de habilidades de procesamiento por diferentes sentidos y cuya importancia por parte del docente es la de proporcionar una idea generalizada para adaptar la clase virtual, donde el conocimiento trasmitido sea el adecuado a la hora de llevarlo a cabo a la práctica profesional, no solo eso, también pude beneficiar al desarrollo de habilidades nuevas y en palabras de Gardner (1998); Emst-Slavit (2001), citados por Nadal (2015b):

Nos habla también de la importancia de que los aprendizajes sean funcionales -es decir, que sirvan para la vida- motivo por el cual considera básico educar para la comprensión, entendida ésta como la capacidad para aplicar los conocimientos y las habilidades adquiridas a nuevas situaciones. En este sentido debemos tener presente que la vida requiere diferentes habilidades (sociales, cognitivas, emocionales...) para afrontarse a los retos cambiantes de la sociedad actual, por tanto, es importante desarrollar todas las inteligencias en lugar de centrarse únicamente en la lingüística y la lógicomatemática como hace la educación tradicional (pág. 130). 


\section{Ensayo Original / Original Essay}

Así pues, el aprendizaje socioemocional es de suma importancia para su aplicación a nivel educativo superior, en el cual los estudiantes pueden desarrollar y llevar a la práctica habilidades emocionales y la gestión de estas, logrando una fácil confrontación en las situaciones reales, pero al no ser en un aula de clases presencial, estas habilidades no se fomentan o se llegan a poner en práctica, porque ahora la digitalización es el nuevo recurso tecnológico para clases virtuales, donde la capacidad de interactuar con las emociones a fortalecer está limitada a factores que no permiten su integración tales como agentes distractores, malos hábitos de estudio, sobrecarga de tareas, etc., como lo menciona Castillo (2020):

Los adolescentes hoy en día no están siendo honestos: su única forma de expresar emociones es exclusivamente desde un punto de vista positivo, pero no son capaces de comunicar emociones como el miedo, el enfado, la decepción o la tristeza. Están viviendo un mundo absolutamente parcial, filtrado, como los filtros de Instagram (pág. 27).

Cabe señalar, que las plataformas educativas son una alternativa temporal ante la situación de pandemia actual, sin embargo, son de suma importancia para poner en práctica los procesos de aprendizaje, pero en realidad el docente solo hace uso de estas plataformas como una herramienta tecnológica pedagógica sin dejar de lado que la educación es parte de un proceso complejo aplicado a un contexto físico, destacando que actualmente lo más importante es fomentar la socialización y mantener un equilibrio emocional, dando una importancia hacia habilidades sociales y emocionales, siendo estas necesarias a nivel superior.

Como resultado al aplicar de fondo la educación emocional en el sistema de educación en México, se tendría más herramientas para afrontar las situaciones emocionales por las que normalmente nos causa conflicto en el entorno académico, personal, familiar y social, haciéndolo notar mediante la frustración, enojo, irritabilidad, entre otras emociones negativas, ante tal 
estallido emocional se toman decisiones equivocadas que en vez de beneficiar producen efectos contraproducentes para la salud y el bienestar de los estudiantes o en su caso repercutiendo en el ambiente laboral.

Ahora bien, si al no tener pleno dominio de la conciencia emocional a etapas tempranas de la educación será un mayor reto la implementación de estas habilidades emocionales a un nivel universitario como se hace notar en el aspecto personal y social, actualmente es evidente en estos tiempos de aislamiento social, siendo muy difícil una empatía provocando una disonancia emocional.

\section{Conclusiones}

La educación emocional tiene como principal función desarrollar competencias emocionales las cuales son de importancia para el estudiante en formación académica debido a que permite un aprendizaje emocional de sí mismo en profundidad, también lograra tener mayor autodominio de su persona en los diferentes contextos, digamos, ser un líder capaz de enfrentar situaciones emocionales y laborales, favoreciendo un crecimiento personal y manteniendo habilidades sociales y empatía ante el contexto social.

En cuanto al proceso de enseñanza-aprendizaje virtual es necesario que el docente digital actualice sus conocimientos y propicie nuevas funciones y métodos que día a día tiene que fundamentar en los saberes de conocimiento, también es necesario que este tenga el conocimiento de las diferentes teorías que pueden beneficiar a sus alumnos, entre ellas es la de las inteligencias múltiples para realizar nuevas propuestas educativas que incluyan modalidades de evaluación y permitan ser más accesibles a un proceso de enseñanza aprendizaje virtual.

Al complementar la educación emocional con estrategias didácticas que favorezcan el conocimiento en los alumnos se desarrollaran nuevas habilidades en la formación académica, permitiendo enfrentarse a un mundo 


\section{Ensayo Original / Original Essay}

laboral donde abunda un sinfín de problemas emocionales y las nuevas generaciones puedan hacer frente a estas situaciones de manera más fácil teniendo un repertorio extenso de competencias que favorezcan al desempeño en un área laboral.

El aislamiento social que actualmente pasa la sociedad es un fenómeno que origina problemas en la salud mental y física sobre todo a los estudiantes universitarios al invertir bastantes horas frente dispositivos electrónicos, provocando conductas negativas y enormes cargas de tecnoestrés provocando un desequilibrio emocional, alterando la convivencia entre sus familiares más cercanos resultando una baja autoestima, disminución de habilidades sociales y un menor desempeño en su rendimiento académico, personal y social.

Las plataformas educativas son una herramienta que se implementa de fondo para seguir con las clases a distancia, teniendo en cuenta que ahora los docentes son digitales y las aulas virtuales, el proceso de enseñanza aprendizaje ha evolucionado, los recursos de cada plataforma son únicos y tienen como propósito que los docentes hagan uso de los recursos con los que se cuenta y se actualizan constantemente para brindar procesos de enseñanza-aprendizaje virtuales mejor calidad, que a su vez pueda medir y comprobar resultados notables en las competencias digitales y emocionales por parte de los actores de la educación.

\section{Referencias}

Bisquerra R., \& García, E. (2018a,b). La educación emocional requiere formación del profesorado. Participación Educativa, 5(8), 15-27, eISSN: 1886-5097. Recuperado de:

https://dialnet.unirioja.es/servlet/articulo?codigo $=6785338$

Castillo, R. (2020). Método Ruler: Habilidades para este siglo. Revista de Educación, (390), 27-29, ISSN: 0716-0534. Chile: Ministerio de 
Ensayo Original / Original Essay

Educación.

Jiménez, A. (2020). Inteligencia emocional. En: AEPap (ed.). Congreso de Actualización Pediatría. Madrid, España: Lúa Ediciones 3.0.

López, É. (2005). La educación emocional en la educación infantil. Revista Interuniversitaria de Formación del Profesorado, 19(3), 153-167, eISSN: 0213-8646. Recuperado de:

https://www.redalyc.org/articulo.oa?id=27411927009

Miguel, J. (2020). La educación superior en tiempos de pandemia: una visión desde dentro del proceso formativo. Rlee: Revista Latinoamericana de Estudios Educativos, 50(especial), 13-40, e-ISSN 2448-878X. Recuperado de:

https://doi.org/10.48102/rlee.2020.50.ESPECIAL.95

Nadal, B. $(2015 a, b)$. Las inteligencias múltiples como una estrategia didáctica para atender a la diversidad y aprovechar el potencial de todos los alumnos. Revista nacional e internacional de educación inclusiva, 8(3), 121-136, ISSN: 1889-4208. Recuperado de:

https://revistaeducacioninclusiva.es/index.php/REl/article/view/94

Palomares-Ruiz, A., \& Serrano-Marugán, I. (2016a,b). Influencia de las Competencias Emocionales y Financieras en la Formación Universitaria. Formación Universitaria, 9(5), 25-36, e-ISSN: 07185006. Recuperado de:

http://dx.doi.org/10.4067/S0718-50062016000500004

Soler, J., Aparicio, L., Díaz, O., Escolano, E., \& Rodríguez, A. (coord.). (2016). Inteligencia Emocional y Bienestar II: reflexiones, experiencias profesionales e investigaciones. ISBN: 978-84-608-4847-9. España: Ediciones Universidad de San Jorge.

Suberviola, I. (2020). Aspectos básicos sobre el concepto y puesta en práctica de la coeducación emocional. Foro de educación, 18(1), 189-207, e-ISSN: 1698-7802. Recuperado de: 
http://dx.doi.org/10.14516/fde.682

World Bank (2020). COVID-19: Impacto en la Educación y Respuestas de Política Pública. Resumen ejecutivo. Washington, DC., Estados Unidos: World Bank. Recuperado de: http://hdl.handle.net/10986/33696 Zepeda-Hernández, S., Abascal-Mena, R., \& López-Ornelas, E. (2015).

Emociones: Factor de Cambio en el Aprendizaje. Ra Ximhai, 11(4), 189-199, e-ISSN: 1665-0441. Recuperado de:

http://revistas.unam.mx/index.php/rxm/article/view/71313 
Ensayo Original / Original Essay

Diego Alvarado Melitón

e-mail: ocupacional fray@outlook.com

Nacido en el estado de México, el 12 de marzo del año

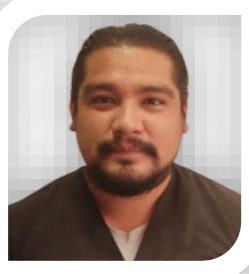

1989. Egresado de la Universidad Autónoma del estado de México (UAEM), con el título de Licenciado en Terapia Ocupacional; actualmente me desempeño como Licenciado en Terapia Ocupacional en el manejo de pacientes psiquiátricos del Hospital Psiquiátrico Fray

El contenido de este manuscrito se difunde bajo una Licencia de Creative Commons ReconocimientoNoComercial-Compartirlgual 4.0 Internacional 